CORRECTION

https://doi.org/10.1038/s41586-019-1017-6

\title{
Author Correction: Hundred-fold enhancement in far-field radiative heat transfer over the blackbody limit
}

Dakotah Thompson, Linxiao Zhu, Rohith Mittapally, Seid Sadat, Zhen Xing, Patrick McArdle,

M. Mumtaz Qazilbash, Pramod Reddy \& Edgar Meyhofer

Correction to: Nature https://doi.org/10.1038/s41586-018-0480-9, published online 3 September 2018.

We would like to correct the sentence in our Letter "However, both our detailed computational analysis (see Supplementary Fig. 2) and past computations ${ }^{13-15}$ suggest that such enhancements are not anticipated for metallic spheres, and very small increases (by a factor of a few) may be expected for dielectric spheres or metallic cylinders." The work of Fernández-Hurtado et al. ${ }^{1}$ (ref. ${ }^{13}$ in the original Letter) is not limited to the structures described in this statement but also presents a computational study of radiative heat transfer between rectangular dielectric membranes that is consistent with our experimental and computational analysis, and supports our findings that the blackbody limit can be overcome in the far-field. The original Letter has not been corrected online.

1. Fernández-Hurtado, V., Fernández-Domínguez, A. I., Feist, J., García-Vidal, F. J. \& Cuevas, J. C. Super-Planckian far-field radiative heat transfer. Phys. Rev. B 97. 045408 (2018) 